\title{
Kausale Satzverknüpfungen im Deutschen
}

Hardarik Blühdorn **

\begin{abstract}
This article gives an overview of the syntactic, prosodic and semantic behaviour, as well as of the textual function, of causal connectives in present day German. In the first section, text coherence is divided into the domains of spatial, temporal and causal coherence. Spatial and temporal coherence are to a considerable extent encoded by grammatical means, while causal coherence is mainly encoded by lexical means: prepositions, conjunctions and adverbs. In the second section, the most important causal connectives of present day German are described, and their basic syntactic and semantic properties introduced. The third section deals with the linguistic concept of cause, contextualizing it within more general philosophical considerations on causation. The concept of cause is traced back to the underlying concepts of situation and condition. The fourth section is devoted to the distinction between three types of causal connections, labelled as dispositional, epistemic and deontic/illocutionary. Empirically, there are many cases in which a causal connection between two sentences can receive more than one of these readings. The following subsections examine in more detail the syntactic, prosodic and semantic conditions that render epistemic and deontic readings of causal connections possible. The main factors influencing the interpretation are shown
\end{abstract}

* Der vorliegende Aufsatz ist die überarbeitete Fassung eines Gastvortrags, der am 09. März 2006 in der Área de Alemão der Universidade de São Paulo gehalten wurde.

* Der Autor ist wissenschaftlicher Angestellter am Institut für Deutsche Sprache und außerplanmäßiger Professor an der Universität Mannheim. 
to be syntactic, prosodic and informational integration of the connected expressions, definiteness of the cause as well as modal contexts.

Keywords: Text coherence; Causal connectives; Prepositions; Conjunctions; Adverbs; Syntax; Prosody; Semantics; Modality.

Zusammenfassung: Der vorliegende Aufsatz gibt einen Überblick über das syntaktische, prosodische und semantische Verhalten sowie die textuelle Funktion kausaler Konnektoren im heutigen Deutsch. Im ersten Abschnitt wird Textkohärenz in räumliche, zeitliche und kausale Kohärenz unterteilt. Räumliche und zeitliche Kohärenz werden zu einem erheblichen Teil durch grammatische Sprachmittel kodiert, während kausale Kohärenz vor allem durch lexikalische Mittel ausgedrückt wird: durch Präpositionen, Konjunktionen und Adverbien. Im zweiten Abschnitt werden die wichtigsten kausalen Konnektoren des Gegenwartsdeutschen vorgestellt und in ihren syntaktischen und semantischen Haupteigenschaften beschrieben. Der dritte Abschnitt behandelt das linguistische Konzept der Ursache vor dem Hintergrund allgemeinerer philosophischer Reflexionen über Kausalität. Das Konzept der Verursachung wird zurückgeführt auf die zugrundeliegenden Konzepte der Situation und der Bedingung. Der vierte Abschnitt ist der Unterscheidung zwischen drei Arten kausaler Verknüpfungen gewidmet, die als dispositionelle, epistemische und deontisch-illokutionäre bezeichnet werden. Empirisch erlauben kausale Verknüpfungen häufig mehr als eine dieser Lesarten. Die folgenden Unterabschnitte untersuchen im Detail die syntaktischen, prosodischen und semantischen Bedingungen, durch die epistemische und deontische Lesarten kausaler Verknüpfungen möglich werden. Als wichtigste Faktoren, die die Interpretation beeinflussen, werden herausgestellt: syntaktische, prosodische und informationelle Integration der verknüpften Ausdrücke, Definitheit der Ursache sowie modale Umgebungen.

Stichwörter: Textkohärenz; Kausale Konnektoren; Präpositionen; Konjunktionen; Adverbien; Syntax; Prosodie; Semantik; Modalität.

Resumo: Este artigo traz uma visão panorâmica do comportamento sintático, prosódico e semântico, bem como da função textual, dos conectores causais do alemão contemporâneo. No primeiro item, a coerência textual é dividida nos domínios da coerência espacial, temporal e causal. Boa parte da coerência 
espacial e temporal é expressa através de recursos gramaticais, enquanto a coerência causal é expressa principalmente por recursos lexicais: preposições, conjunções e advérbios. No segundo item, apresentam-se os conectores causais mais importantes do alemão contemporâneo e as suas propriedades sintáticas e semânticas gerais. $O$ terceiro item trata do conceito lingüístico de causa, contextualizando-o dentro de reflexões filosóficas mais genéricas sobre a causalidade. O conceito de causa é relacionado aos conceitos subjacentes de situação e condição. O quarto item é dedicado à distinção entre três tipos de conexões causais, chamadas de disposicionais, epistêmicas e deônticoilocucionais. Empiricamente, há muitos casos nos quais uma conexão causal entre duas orações pode receber mais do que uma dessas leituras. Os subitens que se seguem examinam, de uma maneira mais detalhada, as condições sintáticas, prosódicas e semânticas que possibilitam leituras epistêmicas e deônticas de conexões causais. Como os principais fatores que influenciam a interpretação destacam-se a integração sintática, prosódica e informacional das expressões conectadas, a definitude da causa, bem como contextos modais.

Palavras-chave: Coerência textual; Conectores causais; Preposições; Conjunções; Advérbios; Sintaxe; Semântica; Prosódia; Modalidade.

\section{Räumliche, zeitliche und kausale Kohärenz im Text}

Einer der Kernbegriffe der Textlinguistik ist der Begriff der Kohärenz. Darunter wird der innere Zusammenhalt eines Textes verstanden, der den Interpreten in die Lage versetzt, dem Text Sinn zuzuschreiben. Allerdings scheint es so zu sein, dass die Begriffe Kohärenz und Sinnzuschreibung sich gewissermaßen gegenseitig erklären: Einem Text kann dann erfolgreich Sinn zugeschrieben werden, wenn er kohärent ist, und ein Text ist dann kohärent, wenn es gelingt, ihm Sinn zuzuschreiben.

Das Gesamtfeld der Kohärenz kann unter verschiedenen Blickwinkeln untersucht werden. Dazu gibt auch heute noch das Buch von HaLLIDAY \& HASAN (1976) einen ausgezeichneten Überblick. Bekannt sind Untersuchungen zu den Referenten, von denen im Text die Rede ist, zu ihrer Neueinführung und referentiellen Wiederaufnahme. Bekannt sind auch Untersuchungen zur räumlichen und zeitlichen Kohärenz von Texten, also zur wechselseitigen Anordnung der Textreferenten relativ zueinander im 
Raum und zur Anordnung der besprochenen Sachverhalte und Ereignisse in der Zeit. Einen guten Überblick über diese Gebiete der Kohärenzforschung gibt VATER (2001 und 2005).

Man kann sagen, dass räumliche und zeitliche Kohärenz, wenn wir hierunter auch die Kohärenz der räumlichen und zeitlichen Referenten, also der besprochenen Personen, Gegenstände und Sachverhalte subsumieren, die Grundlage eines jeden Textverstehens bilden. Wer nicht versteht, von welchen Personen und Dingen im Text die Rede ist und wie diese im Raum angeordnet sind, wer nicht weiß, von welchen Ereignissen und $\mathrm{Zu}-$ ständen der Text handelt und wie diese in der Zeit angeordnet sind, der kann sich keine Hoffnung machen, dem Text irgendeinen Sinn zuzuschreiben, für den muss der Text unvermeidlich ein Rätsel bleiben.

Räumliche und zeitliche Verknüpfungen werden zu einem erheblichen Teil durch obligatorische Sprachmittel kodiert, ohne die kein grammatisch korrekter, vollständiger Satz gebildet werden kann. Für zeitliche Verknüpfungen sind dies die sogenannten TMA-Mittel, also Tempus, Modus und Aspekt. Sie werden grammatisch am Verb ausgedrückt. Räumliche Verknüpfungen werden durch Sprachmittel kodiert, die morphologisch an referentiellen Nominalgruppen ausgedrückt werden. Man kann sie als DCQMittel zusammenfassen: Determinantien (Artikelwörter und Pronomen), Kasus (Case) und Quantifikatoren (vgl. BLÜHDORN 2002). ${ }^{1}$ Es ist kein Zufall,

1 Die Grammatikschreibung hat die Funktion der DCQ-Mittel für die räumliche Kohärenz bis jetzt weniger deutlich herausgearbeitet als die Funktion der TMAMittel für die zeitliche Kohärenz. Unter den Determinantien wird die Funktion der räumlichen Verknüpfung besonders bei den Demonstrativa deutlich, die in vielen Sprachen eine Opposition zwischen räumlicher Nähe und räumlicher Ferne kodieren (im Deutschen dieser vs. jener). Kasus wie Lokativ und Ablativ dienen in Sprachen mit ausgebautem Kasussystem zur Kodierung räumlicher Relationen. Im deutschen Kasussystem ist die Opposition zwischen statischem Dativ (Ortsbestimmung) und dynamischem Akkusativ (Zielbestimmung) bei den sogenannten Wechselpräpositionen bekannt. Auch der Genitiv hat in lexikalisierten Redewendungen noch vereinzelt räumliche Semantik (z.B. einige Kinder kamen des Wegs). Quantifikatoren kodieren unter anderem die Unterscheidung zwischen räumlich begrenzten Individuen und räumlich unbegrenzten Kontinua, die funktional der Aspektualität beim Verb entspricht: 
dass gerade diese Sprachmittel (bzw. zumindest jeweils ein Teil von ihnen) übereinzelsprachlich obligatorisch sind, dass es ohne sie unmöglich ist, grammatisch korrekte und vollständige Sätze zu bilden, denn räumliche und zeitliche Kohärenz bilden die Grundlage, ohne die ein Textverstehen von vornherein ausgeschlossen wäre.

Allerdings sind räumliche und zeitliche Kohärenz für ein angemessenes Textverstehen nur selten ausreichend. Meist ist es notwendig, nicht nur zu verstehen, was geschieht und mit wem es geschieht, sondern man muss auch verstehen, warum es geschieht, um dem Text einen befriedigenden Sinn zuordnen zu können. Dieses Teilgebiet der Kohärenz, das wir als kausale Kohärenz bezeichnen können, ist noch deutlich weniger erforscht.

Auch manche Kausalverknüpfungen müssen in der Sprache obligatorisch kodiert werden. Eine kausale Beziehung besteht z.B. zwischen einem Ereignis und seinem Agens. Viele Sprachen ordnen Agentien standardmäßig die Subjektrolle im Satz zu, die grammatisch immer realisiert sein muss. Trotzdem werden kausale Verknüpfungen in Texten insgesamt weniger explizit gemacht als räumliche und zeitliche. So gibt es in allen Sprachen Möglichkeiten, Sätze ohne Agens zu bilden.

Die kausale Kohärenz, die Frage, warum und wozu die Ereignisse und Zustände eintreten, von denen im Text die Rede ist, betrifft Fragen des vertiefteren Textverstehens. Man kann sagen, dass in der Beantwortung solcher Fragen das Textverstehen gewissermaßen zur Vollendung kommt. Viele dafür relevante Informationen werden im Text nicht explizit kodiert, sondern müssen vom Interpreten durch Inferenzen erschlossen werden. Das liegt daran, dass auch der Textproduzent häufig nicht über das betreffende Wissen verfügt und dass es (deshalb oder aus anderen Gründen) oft nicht erwünscht ist, den Text von vornherein auf eine bestimmte kausale Deutung festzulegen. Ein Textproduzent wird nur dort explizite kausale Verknüpfungen herstellen, wo er eine solche Festlegung für relevant hält. Ansonsten bleibt die Herstellung kausaler Kohärenz und damit die letzte

im Deutschen Brot (räumlich unbegrenzt) vs. ein Brot (räumlich begrenzt). Auch die Unterscheidung zwischen Einzahl und Mehrzahl (ein Brot vs. mehrere Brote) hat räumliche Implikationen (vgl. BLÜHDORN 2006). 
Etappe des Textverstehens und der Sinnzuschreibung dem Interpreten überlassen. So erklärt und rechtfertigt es sich, dass mehrere Interpreten den gleichen Text oft unterschiedlich verstehen.

\section{Sprachmittel für die Kodierung kausaler Verknüpfungen}

Die wichtigsten Sprachmittel, mit denen kausale Verknüpfungen im Text angezeigt werden, sind Präpositionen, Adverbien und Konjunktionen. Auch für die Kodierung räumlicher und zeitlicher Verknüpfungen stehen diese lexikalischen Mittel zusätzlich zu den obligatorischen grammatischen Mitteln zur Verfügung. Aber kausale Verknüpfungen werden im Deutschen und in anderen Sprachen überwiegend durch diese lexikalischen Mittel angezeigt.

Bei der kausalen Kohärenz geht es um Ursachen, Gründe und Motive:

(1) Durch den Regen wurde die Straße unpassierbar.

(2) Wegen seiner Unzuverlässigkeit vertraut dem Minister niemand.

(3) Aus Verzweiflung nahm Maria sich das Leben.

In Beispiel (1) wird der Regen als Ursache für die Unpassierbarkeit der Straße angeführt; in Beispiel (2) wird als Grund für das allgemeine Misstrauen dem Minister gegenüber dessen Unzuverlässigkeit genannt; in Beispiel (3) ist Verzweiflung das Motiv für Marias Selbstmord.

Die Kausalverknüpfungen werden in den Beispielen durch die Präpositionen wegen, durch und aus kodiert. Diese verlangen, wie alle Präpositionen, Nominalgruppen als Ergänzungen (vgl. PASCH et al. 2003: 351). Die Ergänzung einer Präposition bezeichnet das Bezugsobjekt, zu dem die Präposition eine Verknüpfung herstellt. In Beispiel (1) wird erklärt, warum die Straße unpassierbar wurde. Die Erklärung lautet: durch den Regen. Die Präposition durch stellt die Verknüpfung zu dem Bezugsereignis her, auf das die Nominalgruppe den Regen referiert, und weist diesem Ereignis die Relationsrolle URSACHE zu. Der Regen wird also als das verursachende, die Unpassierbarkeit der Straße als das verursachte Ereignis vorgestellt.

Entsprechend ist in Beispiel (2) die Unzuverlässigkeit des Ministers das verursachende und das Misstrauen der Leute das verursachte Ereignis. 
In (3) ist Marias Verzweiflung das verursachende und der Selbstmord das verursachte Ereignis.

Häufige kausale Adverbien sind deshalb, deswegen, daher, also, so, somit und folglich. Wie alle Adverbien werden sie syntaktisch nicht ergänzt, denn Adverbien sind intransitiv, können also keine syntaktischen Ergänzungen zu sich nehmen. Bei ihnen muss der Interpret das Bezugsereignis im Kontext finden, gewöhnlich im vorhergehenden Satz. Zu dem Ausdruck für das Bezugsereignis stellt das kausale Adverb keine syntaktische, sondern lediglich eine semantisch-referentielle Verknüpfung her:

(4) Meine Mutter schlägt mich! Deshalb/deswegen habe ich oft Kopfschmerzen und Schlafstörungen.

(5) Pflanzen haben die Stoffe, die sie zum Überleben brauchen, überall zur Verfügung. Daher/folglich/so brauchen sie sich nicht fortzubewegen.

(6) Es gibt jede Menge Kinderhotels mit allem möglichen Animationsprogramm und sonstigem Kinder-Trara. Also ist es nur recht und billig, wenn es auch das Gegenteil gibt. So dachte es sich ein Hotelier vom Wolfgangsee und erklärte sein Vier-Sterne-Haus zur kinderfreien Zone. (http:/ /www.faz.net - 17.06.2006)

(7) Er hat Hunden gegenüber Aggressionen gezeigt. Somit müssen wir ihn als gefährlich einstufen.

In Beispiel (4) fungieren die Schläge der Mutter als Bezugsereignis mit der Relationsrolle UrSACHE für die Kopfschmerzen und Schlafstörungen. Auch in den Beispielen (5), (6) und (7) liefert jeweils der erste Satz das Bezugsereignis, dem die kausalen Adverbien die Relationsrolle GRUND (Evidenz) (in (5) und (6)) bzw. Motiv (in (7)) zuweisen und mit dem sie jeweils den zweiten Satz semantisch-referentiell verknüpfen.

Die wichtigsten kausalen Konjunktionen sind weil, da und denn. Sie werden, durch Sätze ergänzt, die das Bezugsereignis angeben (vgl. PAsCH et al. 2003: 351). Kausales da ist eine subordinierende Konjunktion (ein Subjunktor), leitet also einen Nebensatz ein; denn ist eine koordinierende Konjunktion (ein Konjunktor), leitet also einen Hauptsatz ein. Weil ist 
standardsprachlich subordinierend wie $d a$, wird aber in der gesprochenen Sprache auch häufig koordinierend wie denn verwendet (zur Syntax von weil vgl. UHMANN 1998):

(8) Gestern war ein langweiliger Tag. Wir konnten nicht an den Strand gehen, denn das Wetter war schlecht.

(9) Warum geht ihr nicht an den Strand? - Wir bleiben lieber im Hotel, weil das Wetter so schlecht ist.

(10) Es regnete den ganzen Tag. Da das Wetter so schlecht war, konnten wir nicht an den Strand gehen.

(11) Wie war's in Italien? - Langweilig. Wir konnten nicht an den Strand gehen, weil das Wetter war schlecht. (gesprochene Sprache)

Die kausalen Konjunktionen weil, da und denn verknüpfen zwei Sätze, von denen der eine die Relationsrolle Ursache, Grund (Evidenz) oder Motiv erhält und der andere die Relationsrolle Wirkung oder Folge(Rung). Sie unterscheiden sich hinsichtlich der Annahmen des Sprechers über das Vorwissen des Adressaten.

Denn verknüpft zwei Sätze, deren Inhalte nach Ansicht des Sprechers für den Adressaten beide neu sind. So nimmt in Beispiel (8) der Sprecher an, dass der Adressat noch nicht weiß, dass man nicht an den Strand gehen konnte, und ebenfalls noch nicht weiß, dass das Wetter schlecht war. Nimmt der Sprecher an, dass eine von beiden Informationen dem Adressaten schon bekannt ist, so kann er die Verknüpfung nicht mit denn herstellen, sondern muss weil oder da wählen.

Auch in Verknüpfungen mit dem Subjunktor weil können Ursache und Wirkung dem Adressaten beide neu sein. Hier ist es aber auch gut möglich, dass die Wirkung schon bekannt und nur die Ursache neu ist. Deshalb kann man mit dem Subjunktor weil sehr gut auf warum-Fragen antworten, was mit denn nicht möglich ist. In Beispiel (9) zeigt die warumFrage, dass der Adressat die Wirkung schon kennt: Der Sprecher und seine Begleiter sind nicht an den Strand gegangen. Nun möchte er den Grund dafür wissen, und dieser wird ihm mit dem weil-Satz mitgeteilt. 
Bei dem Subjunktor da ist es gerade umgekehrt. Er signalisiert die Annahme, dass dem Adressaten die Ursache schon bekannt ist, dass er aber noch nicht weiß, zu welcher Wirkung sie führt oder geführt hat. Deshalb kann man mit da nicht auf warum-Fragen antworten (vgl.: ZIFONUN et al. 1997: 2299f.; Duden 1998: 790). Beispiel (10) zeigt, dass zuerst im Text von dem schlechten Wetter die Rede ist. Zu dem Zeitpunkt, zu dem der Sprecher den $d a$-Satz ausspricht, kann er sicher sein, dass diese Information für den Adressaten nicht neu ist. Neu ist aber die Wirkung, dass man nicht an den Strand gehen konnte, von der der Hauptsatz berichtet.

Wird weil in der gesprochenen Sprache als Konjunktor verwendet, wie in Beispiel (11), so verliert es die Fähigkeit, auf eine warum-Frage zu antworten. In diesem Fall verhält es sich genau wie denn, das heißt, es wird angenommen, dass sowohl die Ursache als auch die Wirkung für den Adressaten neu sind. Weil ist also, wie man sieht, die kausale Konjunktion mit der vielfältigsten Verwendbarkeit. Sie ist in der deutschen Gegenwartssprache bei weitem die häufigste Kausalkonjunktion. Denn und da sind demgegenüber spezialisierter und entsprechend seltener.

Die kausalen Adverbien daher, also, so, somit und folglich verhalten sich ähnlich wie da. Bei ihnen muss zuerst die Ursache genannt werden. An der Stelle, wo diese Adverbien stehen, muss die Ursache dem Adressaten schon bekannt sein, und nur die Wirkung ist noch neu. Man kann diese Adverbien deshalb auch als anaphorisch (rückwärts verweisend) bezeichnen. Die Adverbien deshalb und deswegen können ebenfalls anaphorisch verwendet werden. Sie erlauben aber auch eine vorwärts verweisende (kataphorische) Verwendung, bei der die Ursache erst nachträglich mit weil angeschlossen wird:

(12) Wir sind deshalb/deswegen nicht an den Strand gegangen, weil es regnete.

In solchen Fällen muss dem Adressaten an der Stelle, wo das Adverb steht, die Wirkung bzw. Folge schon bekannt sein. In Beispiel (12) muss er also schon wissen, dass der Sprecher und seine Begleiter nicht am Strand waren. Dagegen ist die Ursache hier neu. Kataphorische Verwendung ist bei den übrigen kausalen Adverbien nicht möglich. 
Als kausaler Konnektor wird häufig auch die adverbiale Partikel nämlich eingestuft:

(13) Ich konnte letzte Woche nicht in die Vorlesung kommen. Ich war nämlich krank.

Hier trägt der erste Satz die Relationsrolle FOLGE und der zweite die Relationsrolle Mотіv. Nämlich ist wiederum anaphorisch, aber es verhält sich konvers zu den anaphorischen kausalen Adverbien. Während kausale Adverbien immer in demjenigen Teilsatz der Verknüpfung stehen, der die Relationsrolle WirkUNG bzw. FOLGE(RUNG) trägt, steht nämlich in dem Teilsatz, der die Relationsrolle URSACHE, GRUND oder MotTV trägt. Es ist deshalb konsequenter, nämlich nicht als Kausal-, sondern als Konsekutivkonnektor einzustufen.

\section{Was ist unter Kausalität zu verstehen?}

Ich möchte nun ein wenig genauer der Frage nachgehen, was eigentlich mit kausalen Verknüpfungen ausgesagt wird. In Wolfgang STEGMÜLLERs klassischem Aufsatz Das Problem der Kausalität (1960=1974: 17) heißt es:

„(...) so würde ein heutiger Physiker nicht nur leugnen, daß es zu jedem Ereignis eine adäquate kausale Erklärung gibt, sondern vermutlich die wesentlich schärfere Behauptung aufstellen, daß für kein Ereignis eine wissenschaftlich haltbare kausale Erklärung existiert."

Ginge es um Kausalität in der außersprachlichen Welt, so könnten wir unsere Überlegungen hiermit beenden. Als Sprachwissenschaftler jedoch interessieren wir uns nicht für Kausalverknüpfungen zwischen Ereignissen, sondern für Kausalverknüpfungen zwischen Sätzen. Was aber ist im linguistischen Sinne unter Ursache und Wirkung zu verstehen?

Bei Stegmüller (1960=1974: 4f.) findet sich die folgende Passage, die ich leicht modifiziert wiedergebe: 
Angenommen, ein Haus wird dadurch zum Einsturz gebracht, dass im Verlaufe von Bauarbeiten im Keller eine Stütze entfernt wird. Es wird dann gesagt, dass die Entfernung jener Stütze die Ursache für den Einsturz des Hauses war. Es ist aber klar, dass das Haus trotz der Entfernung der Stütze keineswegs eingestürzt wäre, wenn es eine andere Beschaffenheit gehabt hätte. Wenn z.B. die Struktur des Hauses eine solche gewesen wäre, dass die Aufrechterhaltung seiner Stabilität die Stütze nicht erfordert hätte, so wäre weiter gar nichts geschehen. Wenn wir also die Beseitigung jener Stütze als die Ursache für den Einsturz bezeichnen, so ist dies im Grunde eine vollkommen einseitige Beschreibung des Vorganges. Die Beseitigung der Stütze muß in Wahrheit mit einer großen Anzahl von anderen Faktoren zusammentreffen, um den Einsturz des Hauses hervorzurufen. Diese anderen Faktoren aber wurden überhaupt nicht erwähnt.

Was diese Überlegung deutlich macht, ist die hochgradige Selektivität alltäglicher Kausalerklärungen. Aus einer Vielzahl von Faktoren, die zusammenspielen müssen, damit ein bestimmtes Ereignis eintritt, wird, je nach aktuellem Interesse, ein einziger ausgewählt und dem Ereignis als Ursache zugeordnet. Die Einzigkeit und Alleinverantwortlichkeit dieses Faktors wird dabei in der Regel nicht behauptet. Die Auswahl erfolgt vielmehr aufgrund der rein pragmatischen Beschränkung, dass man gar nicht alle Faktoren kennt, die in der Situation mitspielen, und/oder dass es nicht relevant wäre, sie allesamt aufzuzählen.

Ursache im linguistischen Sinne ist also nicht das, was in der außersprachlichen Welt eine Wirkung hervorbringt, sondern das, was mittels sprachlicher Markierung einer Wirkung als Ursache zugeordnet wird, das, was sprachlich als Ursache ausgewiesen wird. Nach STEGMÜLLER (1960=1974: 17f.) kann Verursachung außersprachlich gar nicht nachgewiesen werden. Sie kann lediglich sprachlich behauptet und dann geglaubt oder nicht geglaubt werden.

Die Ausweisung von Ursachen im Alltag folgt häufig dem Prinzip post hoc ergo propter hoc (,danach, also aus diesem Grunde), das in der Logik als Fehlschluss eingestuft wird (vgl. KONDAKOw 1983: 387). Sprachlich hat sich dieses Prinzip in der Etymologie mancher Kausalmarker verfestigt. So stammt der kausale Subjunktor weil von dem temporalen Substantiv die Weile 
ab; der kausale Konjunktor denn geht auf das temporale Adverb denn/dann zurück. Allerdings liegen die Ursprünge der meisten Kausalmarker nicht in zeitsemantischen, sondern in raumsemantischen Ausdrücken: so ist wegen abgeleitet von dem Substantiv der Weg, infolge von dem Verb folgen (dessen zeitliche Bedeutung wiederum auf eine räumliche zurückgeht), da ist zunächst ein Raumadverb, durch eine Raumpräposition, halb (wie in deshalb) und halber bedeuten ursprünglich ,räumlich danebenliegend', wie die eine Hälfte neben der anderen (vgl. insgesamt PFEIFER et al. 1989).

Dahinter steckt eine allgemeinere Stufenfolge von drei Betrachtungsweisen, die ich als die situierende, die konditionale und die kausale bezeichnen möchte (vgl. BLÜHDORN 2003: 19f.). Schauen wir dazu folgende Beispiele an:

(14) Wo der Eingang liegt, ist die Tapete rot.

(15) Wo das Loch ist, kann ich nicht fahren.

In beiden Fällen geht es um die Kontiguität (hier die räumliche Angrenzung) zweier Sachverhalte: An einer bestimmten Stelle des Raumes liegt der Eingang, und da ist die Tapete rot; an einer bestimmten Stelle der Fahrbahn ist ein Loch, und da kann der Sprecher nicht fahren. Beide Sachverhalte können gänzlich unabhängig voneinander sein. So ist es vermutlich in Beispiel (14). In Beispiel (15) wird allerdings der Sachverhalt im Nachsatz durch die Modalisierung auf Bedingungen bezogen: kann nicht bedeutet, es bestehen hinreichende Gegenbedingungen'. Es liegt nahe, den im Vordersatz beschriebenen Sachverhalt als eine der relevanten Gegenbedingungen zu deuten. Damit findet ein Übergang von der (räumlich) situierenden zur konditionalen Betrachtungsweise statt.

Bedingungen können das Eintreten von Folgen in unterschiedlichem Grade begünstigen oder behindern. Dadurch erhalten die Folgen modale Werte: Möglichkeit, Notwendigkeit, Beliebigkeit oder Unmöglichkeit. Darüber hinaus können Folgen Faktizitätswerte annehmen: Was notwendig, möglich oder beliebig ist, kann einen positiven Faktizitätswert erhalten, d.h. eintreten. Was unmöglich, möglich oder beliebig ist, kann einen negativen Faktizitätswert erhalten, d.h. nicht eintreten. Folgen, denen ein positiver oder negativer Faktizitätswert zugeordnet ist, heißen Wirkungen; die für 
ihren Faktizitätswert herangezogenen Bedingungen heißen Ursachen. Das Loch in Beispiel (15) ist nicht nur eine hinreichende Gegenbedingung für das Fahren, sondern es ist auch eine Ursache für dessen faktisches NichtEintreten: Ich kann da nicht fahren, wenn da ein Loch ist, und ich fahre da nicht, weil da das Loch ist. Damit findet ein Übergang von der konditionalen zur kausalen Betrachtungsweise statt.

\section{Drei Lesarten kausaler Verknüpfungen}

Nach Sweetser (1990: 76ff.) können kausale Verknüpfungen auf unterschiedliche Arten gelesen werden. Einem Beispielsatz wie:

(16) Peter bleibt zu /HAU \se, // weil es so stark /REG \net. ${ }^{2}$

können mindestens die drei folgenden Paraphrasen zugeordnet werden:

(16a) Peter bleibt zu Hause, und der Grund dafür ist die Tatsache, dass es so stark regnet.

(16b) Ich bin überzeugt davon, dass Peter zu Hause bleibt, und der Grund für diese Überzeugung ist mein Wissen um die Tatsache, dass es so stark regnet.

(16c) Ich ordne an, dass Peter zu Hause bleibt, und der Grund für diese Anordnung ist meine Bewertung der Tatsache, dass es so stark regnet.

(16a) nenne ich die dispositionelle, (16b) die epistemische und (16c) die deontische Lesart des Satzes. Der Hauptunterschied zwischen diesen Lesar-

2 Akzentsilben werden durch Großbuchstaben hervorgehoben. Neben- bzw. Topikakzent (vgl. BürING 1997, MORONI 2006) wird durch einen steigenden Schrägstrich vor der betreffenden Silbe, Haupt- bzw. Fokusakzent durch einen fallenden Schrägstrich (backslash) nach der Silbe angezeigt. Gibt es nur eine Silbe mit Satzakzent, so steht der steigende Schrägstrich vor und der fallende nach dieser Silbe. Grenzen zwischen Intonationsgruppen werden durch doppelte Schrägstriche markiert. 
ten liegt in der Verknüpfungsdomäne, in der der Konnektor weil verrechnet wird. In (16a) operiert er auf der Ebene der Sachverhalte, d.h. er bestimmt einen Sachverhalt als Wirkung und einen anderen als dazugehörige Ursache. Andere Autoren sprechen in diesem Zusammenhang auch von Realgründen (vgl. ZifOnUn et al. 1997: 2296ff.). In (16b) operiert er auf der Ebene wahrheitsbewerteter Propositionen. Im vorliegenden Fall verknüpft er eine Schlussfolgerung des Sprechers mit einer Evidenz, die die Schlussfolgerung stützt. In der Literatur wird auch von Erkenntnisgründen gesprochen (vgl. ebd.). In (16c) operiert er auf der Ebene der Akte. Das sind Objekte, die bezüglich ihrer Erwünschtheit bewertet werden (vgl. BLÜHDORN 2003: 17). Im Beispiel geht es um eine Anordnung und um ein Motiv, das die Anordnung rechtfertigt. Diese Lesart wird in der Literatur auch als die illokutionäre bezeichnet (vgl. Sweetser 1990: 77; Keller 1995: 23f.). Entsprechend den drei Verknüpfungsebenen können wir der Konjunktion weil eine dispositionelle, eine epistemische und eine deontisch-illokutionäre Lesart zuordnen. Die dispositionelle Lesart ist nicht-modal, die epistemische und die deontische sind modal.

URSACHE, GRUnd und Motiv sind demnach drei Varianten einer abstrakten Relationsrolle, die wir vERURSACHENDES OBJEKT nennen können und die in allen Kausalverknüpfungen vorkommt. URSACHE heißt das verursachende Objekt in einer dispositionellen Kausalverknüpfung, GRUND (bzw. Evidenz) heißt es in einer epistemischen Kausalverknüpfung und Mотіv heißt es in einer deontisch-illokutionären Kausalverknüpfung. Ebenso sind WirkUng und Folge(RUNG) Varianten der abstrakten Relationsrolle verursachtes OBjekt. Wirkung heißt diese Relationsrolle in dispositionellen Kausalverknüpfungen, Folge(RUNG) heißt sie in modalen Kausalverknüpfungen.

\subsection{Wann sind modale Lesarten der Kausalverknüpfung möglich?}

Nicht jede kausale Satzverknüpfung erlaubt alle drei Lesarten des Konnektors. Vergleichen wir unseren Beispielsatz (16) mit der syntaktischen Umkehrung (17): 
(16) Peter bleibt zu /HAU \se, // weil es so stark /REG \net.

(17) Weil es so stark /REGnet, bleibt Peter zu HAU \se.

Wenn der kausale Nebensatz im Vorfeld steht, ist die epistemische Deutung im Sinne der Paraphrase (16b) nicht verfügbar. (17) kann also nur bedeuten: ,es regnet so stark, und deshalb bleibt Peter zu Hause', nicht aber: ,ich weiß, dass es so stark regnet, und deshalb bin ich überzeugt davon, dass Peter zu Hause bleibt‘. Dahinter steckt ein Skopus-Unterschied, den wir uns folgendermaßen klarmachen können.

Nach JACOBS (1984: 32ff.) gehört zur semantischen Repräsentation eines Satzes außer der Proposition ein modaler Operator, der IllokutionsInformation einbringt. Analog werde ich einen epistemischen Operator annehmen, der den Grad an Sicherheit ausdrückt, den der Sprecher der Proposition zuordnet (vgl. auch PASCH et al. 2003: 163ff.). So kann Satz (18) vereinfacht in die Formel (18a) übersetzt werden:

(18) Peter bleibt zu Hause.

(18a) CERT(bleib-(zu Hause, Peter))

Der Operator CERT steht für die unmarkierte epistemische Modalität, in der keine Zweifel an der Wahrheit der geäußerten Proposition im intendierten Referenzbereich angezeigt werden. Er ist dem Prädikat bleibübergeordnet. Für Satz (16) ergeben sich nun zwei unterschiedliche semantische Strukturen, die den Lesarten (16a) und (16b) entsprechen:

(19a) CERT(WEIL(es so stark regnet), bleib-(zu Hause, Peter))

(19b) WEIL(CERT(es so stark regnet), CERT(bleib-(zu Hause, Peter)))

In Struktur (19a) hat der Operator CERT Skopus über den Konnektor WEIL. Dieser verknüpft hier zwei Sachverhalte. Das entspricht seiner dispositionellen Lesart (16a). In Struktur (19b) dagegen hat WEIL Skopus über die modalen Operatoren der Teilsätze. Hier ist jedem Teilsatz ein eigener modaler Operator zugeordnet, im vorliegenden Fall beidemal CERT. Der Konnektor verknüpft zwei Propositionen samt ih- 
ren modalen Operatoren miteinander. Das entspricht seiner epistemischen Lesart (16b).

Damit ein Konnektor modal gelesen wird, müssen seine Konnekte Propositionen sein. Ferner muss jedem seiner Konnekte ein eigener modaler Operator zugeordnet sein, und beide Operatoren müssen im Skopus des Konnektors stehen (vgl. PAscH et al. 2003: 175ff.). Damit diese semantische Konstellation zustande kommt, darf der Konnektor syntaktisch nicht Konstituente beider Teilsätze sein, darf also zum Beispiel nicht zusammen mit dem Nebensatz im Vorfeld des Hauptsatzes stehen wie in (17) (zur Syntax der deutschen Kausalkonjunktionen vgl. Uhmann 1998).

Betrachten wir eine Beispielgruppe, an der der Zusammenhang zwischen syntaktischer Anordnung und modaler Lesart der Kausalverknüpfung ganz deutlich wird:

(20a) Da Sie mich gefragt haben, hat Peter heute keine Zeit.

(20b) Da Sie mich gefragt haben: Peter hat heute keine Zeit.

(20c) Peter hat heute - da Sie mich gefragt haben - keine Zeit.

(20d) Peter hat heute keine Zeit - da Sie mich gefragt haben.

Satz (20a), mit dem kausalen Nebensatz im Vorfeld, kann man nur so interpretieren, dass irgendeine inopportune Frage Ursache dafür ist, dass Peter heute keine Zeit hat, also dispositionell. In (20b) ist der vorangestellte Kausalsatz syntaktisch abgetrennt, steht also nicht im Vorfeld. Im Vorfeld des Nachsatzes steht dessen Subjekt Peter. Hier ist die Kausalverknüpfung modal (deontisch-illokutionär) zu interpretieren: Die Frage ist das Motiv, das den Sprecher zu der Mitteilung veranlasst, dass Peter keine Zeit hat. (20c) und (20d) zeigen, dass die gleiche Interpretation auch bei Mittelfeldund Nachfeld-Stellung des Nebensatzes möglich ist, allerdings nur wenn dieser als Parenthese vom übrigen Satz abgetrennt wird (vgl. PAsCH et al. 2003: 245ff.). Die Generalisierung lautet: Kausale Konnektoren werden bei syntaktischer Integration dispositionell gelesen. Bei syntaktischer Desintegration werden modale Lesarten möglich (vgl. WEGENER 1999: 17f.). 


\subsection{Konnektorklasse und syntaktische Integration}

Diese Verallgemeinerung muss allerdings durch eine Reihe von Zusätzen ergänzt und relativiert werden. Einer von ihnen betrifft die syntaktische Klasse des Konnektors. Subjunktoren wie weil und da können wir einen mittleren Grad an syntaktischer Integrationskraft zusprechen. Ursache und Wirkung werden in zwei Teilsätzen kodiert, von denen der eine in den anderen eingebettet ist (Hypotaxe). Das Mittel der Parenthese gibt uns die Möglichkeit, beide Sätze syntaktisch voneinander zu trennen. Dann kann der Nebensatz als eigenständige kommunikative Minimaleinheit im Sinne von ZIFONUN et al. (1997: 86) fungieren. Seine semantische Repräsentation erhält einen eigenen modalen Operator, und dieser kann zusammen mit dem modalen Operator des syntaktischen Obersatzes in den Skopus des kausalen Konnektors treten.

Höhere syntaktische Integrationskraft besitzen kausale Präpositionen wie wegen. Sie bilden zusammen mit ihren Ergänzungen nicht-satzförmige Konstituenten:

(21) Peter bleibt wegen des starken Regens zu Hause.

Präpositionale Kausalangaben erschweren modale Konnektor-Lesarten, auch in Parenthese:

(21a) Wegen des starken Regens: Peter bleibt zu Hause.

(21b) Peter bleibt - wegen des starken Regens - zu Hause.

(21c) Peter bleibt zu Hause - wegen des starken Regens.

Die Kausalverknüpfungen in (21a) bis (21c) sind trotz ihrer syntaktischen Desintegration bevorzugt dispositionell zu lesen. Das liegt daran, dass sie nur schwer als eigenständige kommunikative Minimaleinheiten gedeutet werden können. Damit kann ihnen auch nur schwer ein eigener modaler Operator zugeordnet werden, der in den Skopus des Konnektors treten könnte. Ist eine modale Interpretation der Kausalverknüpfung in solchen Fällen intendiert, so wird sie durch eine modale Umgebung erleichtert, also etwa durch Modalverben, Modalpartikeln oder modale Adverbien: 
(21d) Peter bleibt wohl zu Hause - wegen des starken Regens. (epistemisch) (21e) Peter muss zu Hause bleiben - wegen des starken Regens. (deontisch)

Geringere syntaktische Integrationskraft als Präpositionen und Subjunktoren besitzen Konjunktoren wie denn und weil mit Verb-Zweit-Satz (vgl. PAsCH et al. 2003: 267ff., 305f.):

(22) Peter bleibt zu /HAU \se, // denn (weil) es /REG \net so stark.

Bei ihnen werden Ursache und Wirkung immer in getrennten Hauptsätzen kodiert (Parataxe), und der Konnektor steht immer im Nachsatz (vgl. Uhmann 1998: 107ff.). Hier können beide Konnekte problemlos als eigenständige kommunikative Minimaleinheiten auftreten, und ihre modalen Operatoren können in den Skopus des Konnektors treten. Deshalb werden denn-Verknüpfungen bevorzugt modal interpretiert, und Rudi Keller konnte das koordinierende weil mit Verb-Zweit-Satz aus demselben Grunde überzeugend als epistemisches weil beschreiben (vgl. KELLER 1995). Allerdings sind modale Konnektor-Lesarten unter diesen Umständen keineswegs obligatorisch. Die Kausalverknüpfung in (22) kann durchaus auch dispositionell verstanden werden. Zwischen getrennten Sätzen können semantische Relationen auf allen Ebenen hergestellt und die modalen Operatoren in der Repräsentation entsprechend eingerichtet werden. Beschränkungen gelten nur bei erhöhter syntaktischer Integration (vgl. BLÜHDORN i.E.).

Adverbkonnektoren stellen überhaupt keine syntaktische, sondern nur eine semantisch-referentielle Verknüpfung zwischen ihren Konnekten her. Zugleich sind sie aber in eines ihrer Konnekte syntaktisch integriert:

(23) Es /REG \net. // Deshalb bleibt Peter zu /HAU \se.

Die meisten Adverbkonnektoren sind wie Konjunktoren auf allen Verknüpfungsebenen interpretierbar. Einige von ihnen weisen aber idiosynkratische Spezialisierungen für bestimmte Verknüpfungsebenen auf. 
So wird der adverbiale Partikelkonnektor nämlich bevorzugt modal interpretiert (vgl. ebd.).

\subsection{Syntax und Prosodie}

Wie die Beispiele (16), (17), (22) und (23) gezeigt haben, hat die syntaktische Struktur auch Konsequenzen in der Prosodie. In parataktischen Verknüpfungen wie (22) und (23) müssen beide Teilsätze stets einen eigenen Hauptakzent tragen (vgl. UHMANN 1998: 127). In hypotaktischen Verknüpfungen können beide Teilsätze einen eigenen Akzent tragen wie in (24a); es kann aber auch ein Teilsatz unakzentuiert bleiben wie in (24b):

(24a) \{Und was macht / PEter?\} Peter bleibt zu /HAU \se, // weil es so stark /REG \net.

(24b) $\{$ Warum bleibt Peter denn heute zи /HAU \se?\} Peter bleibt zu Hause, weil es so stark /REG \net.

Sätze mit präpositionaler Kausalangabe erhalten im Normalfall nur einen Hauptakzent, etwa wie in $(25 \mathrm{a} / \mathrm{b})$ :

(25a) \{Und was macht / PEter?\} / PEter bleibt wegen des starken /REgens zu HAU $\backslash$ se.

(25b) $\{$ Warum bleibt Peter denn beute zu /HAU \se?\} Peter bleibt wegen des starken /RE\gens zu Hause.

Eine modale Interpretation der Kausalverknüpfung ist nur dann ohne weiteres möglich, wenn verursachendes und verursachtes Objekt auf zwei Teilsätze mit eigenem Hauptakzent verteilt sind, also in (22), (23) und (24a) (vgl. SweEtser 1990: 82ff.). Ist nur der kausale Nebensatz akzentuiert und der Hauptsatz unakzentuiert wie in (24b), so kann die Kausalverknüpfung nur dispositionell gelesen werden. Gleiches gilt bei präpositionalen Kausalangaben wie in $(25 \mathrm{a} / \mathrm{b})$.

Nach JaCoBs (1984: 26f.) markieren Hauptakzente den Informationsfokus im Satz, also diejenigen Informationsbestandteile, die im gegebenen 


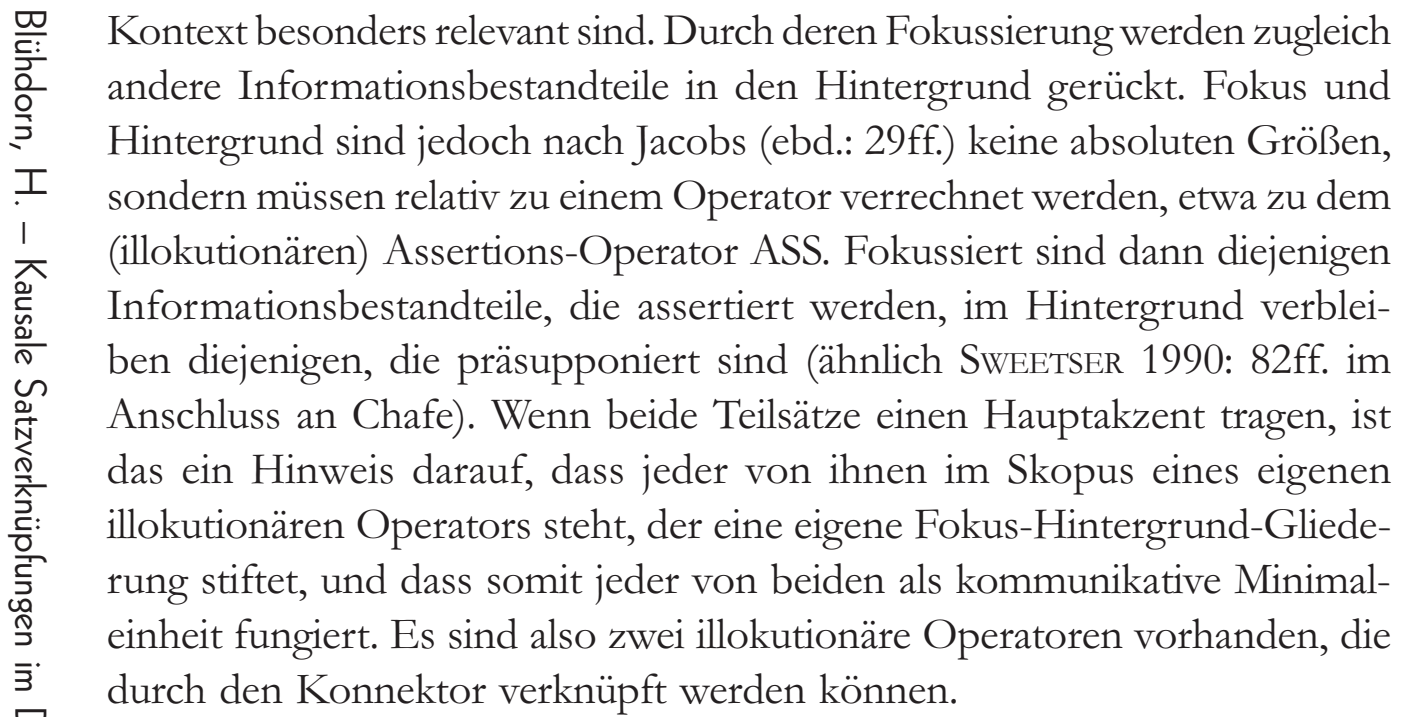

$\Xi$ durch den Konnektor verknüpft werden können.

\subsection{Definitheit der Ursache}

In engem Zusammenhang mit der Fokus-Hintergrund-Gliederung stehen Bekanntheit und Neuheit der Information. Betrachten wir folgendes Satzpaar:

(26) Peter bleibt wegen des Regens zu Hause.

(27) Peter bleibt wegen eines Schauers zu Hause.

In (26) wird das verursachende Objekt (im Folgenden sage ich kurz die Ursache und beziehe mich damit, je nach Kontext, auch auf Gründe und Motive) mit dem Definitartikel als schon bekannter oder im Kontext identifizierbarer Referent eingeführt. In (27) wird die Ursache mit dem Indefinitartikel als neuer, im Kontext noch nicht identifizierbarer Referent eingeführt.

Eine entsprechende Opposition ist im Deutschen, wie wir gesehen haben, auch in hypotaktischen Satzverknüpfungen möglich:

(28) Peter fährt mit dem Bus, da er kein Auto hat.

(29) Peter fährt mit dem Bus, weil er kein Auto hat. 
In der Literatur ist wiederholt festgestellt worden, dass die Konjunktion da typischerweise verwendet wird, wenn die mit ihr eingeführte Ursache im Kontext bereits bekannt ist (vgl. z.B. REDDER 1990: 269ff., 320; WeINRICH 1993: 756ff.; Duden 1998: 789; WegENER 1999: 21f.). Ebenso wie andere $d$-Elemente des Deutschen ist da von Haus aus ein Anzeiger von Definitheit, und zwar in der Kausalrelation von Definitheit der Ursache (vgl. BLÜHDORN 2002: 266f.; BlÜHDORN 2003: 15ff.). Demgegenüber ist weil in dem Sinne indefinit, dass es Bekanntheit oder Identifizierbarkeit der Ursache nicht anzeigt (indefinit $=$ für Definitheit unmarkiert). Wie wir gesehen haben, ist weil der einzige Subjunktor, mit dem man warum-Fragen beantworten kann, mit dem also bei schon bekannter Wirkung noch unbekannte, neue Ursachen eingeführt werden können. Allerdings schließt weil als der am wenigsten spezialisierte Kausalkonnektor auch Verknüpfungen mit schon bekannter Ursache nicht aus.

Als bekannt behandelte Informationsbestandteile sind im Skopus eines Assertions-Operators gute Hintergrund-Kandidaten, weil sie geringen Mitteilungswert haben. Daher gelten für Sätze wie (26) und (27) unterschiedliche Akzentuierungsregeln. Wird auf die Ursache definit referiert, so kann die Kausalangabe unakzentuiert bleiben wie in (26a), einen Nebenakzent erhalten wie in (26b) oder den Hauptakzent tragen wie in (26c):

(26a) \{Und was macht / PEter bei diesem Wetter?\} Peter bleibt wegen des Regens zu /HAU \se.

(26b) \{Und was macht / PEter in dieser Notsituation?\} / PEter bleibt wegen des /REgens zu HAU \se.

(26c) $\{$ Warum bleibt Peter denn $z$ « $/ H A U \backslash s e ?\}$ Peter bleibt wegen des / RE\gens zu Hause.

Dagegen ist für Satz (27) die Akzentuierungsvariante (27a) mit nichtakzentuierter referentieller Kausalangabe prosodisch abweichend. Die indefinit referierende Kausalangabe muss mindestens einen Nebenakzent wie in (27b) oder aber den Hauptakzent wie in (27c) erhalten: 
(27a) \{Und was macht / PEter bei diesem Wetter?\} *Peter bleibt wegen eines Schauers zu /HAU \se.

(27b) \{Und was macht / PEter in dieser Notsituation?\} /PEter bleibt wegen eines /SCHAUers zu HAU \se.

(27c) $\{$ Warum bleibt Peter denn zu /HAU\se?\} Peter bleibt wegen eines / SCHAU \ers zu Hause.

Eine ähnliche Asymmetrie können wir bei kausalen Subjunktor-

Verknüpfungen beobachten:

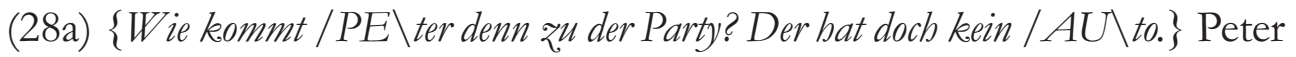
fährt mit dem /BUS $\backslash$, da er kein Auto hat.

(28b) $\{$ Warum fährt Peter denn mit dem / BUS \?\} */PEter fährt mit dem / BUS, da er kein AU to hat.

(28c) \{Und was macht / PEter?\} Peter fährt mit dem /BUS $\backslash, / /$ da er kein / $\mathrm{AU} \backslash$ to hat.

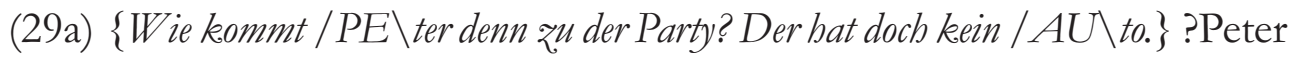
fährt mit dem /BUS \, weil er kein Auto hat.

(29b) \{Warum fährt Peter denn mit dem / BUS $\backslash$ ? $\}$ / PEter fährt mit dem /BUS, weil er kein $A U \backslash$ to hat.

(29c) \{Und was macht / PEter?\} Peter fährt mit dem /BUS $\backslash, / /$ weil er kein /AU to hat.

Nicht-akzentuierte weil-Sätze wie in (29a) klingen, zumindest für mein Sprachgefühl und das mehrerer Informanten, grundsätzlich merkwürdig. In der Literatur werden sie kaum behandelt. Offenbar verlangt weil nach einem Komplement mit einem gewissen kommunikativen Mindestgewicht und lässt entsprechend zumindest einen Nebenakzent auf dem Kausalsatz erwarten. Diese Eigenschaft passt zu seiner Funktion, warum-Fragen zu beantworten. Wo Äußerungen wie (29a) dennoch vorkommen, machen sie den Eindruck stilistisch ungeschickter Formulierungen. 
Nicht-akzentuierte da-Sätze wie in (28a) sind im Prinzip unproblematisch. ${ }^{3}$ Die durch $d a$ angezeigte Bekanntheit der Ursache passt gut mit Nicht-Akzentuiertheit zusammen. Allerdings führen nachgestellte nicht-akzentuierte da-Sätze wie in (28a) zu einer markierten Rhema-ThemaAbfolge, die nur in bestimmten Kontexten sinnvoll ist. Weil-Sätze mit Hauptakzent wie in (29b) und (29c) sind prosodisch unauffällig, unabhängig davon, ob sie kommunikative Minimaleinheiten bilden oder nicht. Dagegen sind da-Sätze mit Hauptakzent nur zulässig, wenn sie eigenständige kommunikative Minimaleinheiten bilden wie in (28c). In diesem Fall kann ihnen die kommunikative Funktion einer Erinnerung an die im Prinzip schon bekannte Ursache zugeordnet werden. Als syntaktisch und kommunikativ integrierte Konstituenten wie in (28b) sind Hauptakzent tragende da-Sätze ungrammatisch. Entsprechend können da-Sätze, wie wir schon gesehen haben, nicht auf warum-Fragen antworten.

Eine wichtige Beobachtung besteht nun darin, dass Satzverknüpfungen mit definitem Kausalsatz unter bestimmten Umständen epistemische Konnektor-Lesarten ermöglichen, die bei indefinitem Konnektor ausgeschlossen sind, und zwar wenn der Kausalsatz im Vorfeld steht (vgl. ZiFOnUn et al. 1997: 2305; auch UhMANN 1998: 126):

(30a) $\{$ Warum / AR beitet er denn noch? $\}$ Da seine /LAM $\backslash$ pe noch brennt, arbeitet er noch.

\footnotetext{
Nicht-akzentuierte Nebensätze mit wo, wenn, als, da und zahlreichen anderen Subjunktoren sind, je nach Kontext, möglich, nicht aber unakzentuierte weilSätze. Um diesen Unterschied zu erklären, ist noch genauere Forschungsarbeit notwendig. Als unmarkiertes Glied der Definitheits-Opposition hat weil von Haus aus einen größeren Verwendungsbereich als das markierte da und kann oft für dieses eintreten. Seine Verwendung wirkt aber stilistisch ungeschickt, wenn der Kontext eindeutig Definitheit fordert und keine warum-Frage zu beantworten ist. In der Gegenwartssprache tendiert weil dazu, die Funktionen von denn und da insgesamt mitzuübernehmen (vgl. Pasch 1997). So wird die Definitheits-Opposition zu da heute von manchen Sprechern nicht mehr beobachtet. Für solche Sprecher besteht möglicherweise kein Unterschied zwischen (28a) und (29a).
} 
(30b) $\{W$ as er wohl gerade /MACHT.$\} \mathrm{Da}$ seine /LAMpe noch brennt, $\mathrm{AR} \backslash$ beitet er noch.

(31a) $\{$ Warum / AR beitet er denn noch?\} Weil seine /LAM $\backslash$ pe noch brennt, arbeitet er noch.

(31b) \{Was er wobl gerade /MACHT $\backslash$. Weil seine /LAMpe noch brennt, $\mathrm{AR} \backslash$ beitet er noch.

Im Vorfeld können Kausalsätze nur dann den Hauptakzent tragen, wenn der nachfolgende Obersatz unakzentuiert bleibt. Dies ist nur mit weil möglich, wie in (31a), nicht aber mit da wie in (30a). Hauptakzent-tragende syntaktisch integrierte $d a$-Sätze sind, wie wir gesehen haben, ungrammatisch. Trägt der Obersatz den Hauptakzent wie in (30b) und (31b), so kann der Kausalsatz im Vorfeld maximal einen Nebenakzent erhalten. Dies ist sowohl mit weil als auch mit da möglich, führt aber zu unterschiedlichen Interpretationsmöglichkeiten.

Bis jetzt haben wir angenommen, dass in Konstruktionen, in denen der Kausalsatz syntaktisch integriert ist, also z.B. im Vorfeld steht, die Kausalverknüpfung nur dispositionell gelesen werden kann. (31b) steht im Einklang mit dieser These. Hier kann die Interpretation nur lauten: ,das Brennen der Lampe veranlasst ihn weiterzuarbeiten' (z.B. weil er das Licht ausnutzen möchte). Eine modale Lesart des Konnektors ist ausgeschlossen. Ist eine epistemische Interpretation dennoch gewollt, so werden im Kontext zusätzliche Hinweise benötigt, die eine solche Lesart nahelegen (vgl. WEGENER 1999: 22).

Auch (30b) kann, im gleichen Sinne wie (31b), dispositionell gelesen werden. Daneben erlaubt es jedoch eine epistemische Interpretation: ,ich sehe, dass seine Lampe noch brennt, und das ist eine Evidenz, die mich davon überzeugt, dass er noch arbeitet' (vgl. ZIFONUN et al. 1997: 2305, die sogar eine dispositionelle Deutung von da ganz ausschließen möchten). Dies muss an der Definitheit von da liegen, die den einzigen Unterschied zwischen (30b) und (31b) bildet.

Modale Konnektor-Lesarten kommen zustande, wenn die modalen Operatoren der Konnekte in den Konnektor-Skopus treten. Nebensätze 
haben nur dann eigene modale Operatoren, wenn sie kommunikative Minimaleinheiten sind. In diesem Fall besitzen sie eine eigene FokusHintergrund-Struktur, tragen einen eigenen Hauptakzent und stehen syntaktisch außerhalb des Hauptsatzes. All das ist in (31b) nicht der Fall. Somit kann die Kausalverknüpfung in (31b) nicht modal interpretiert werden.

Die Definitheit von da aber scheint dieses Regelgefüge teilweise außer Kraft zu setzen, denn auch in (30b) sind die Bedingungen nicht erfüllt. Offenbar kann der Subjunktor da in diesem Beispiel nicht nur einen Ausdruck mit Sachverhaltsreferenz, sondern auch eine Proposition als Komplement nehmen. Wie aber ist dies möglich, ohne dass der Nebensatz eine eigene kommunikative Minimaleinheit bildet? Um dies zu verstehen, müssen wir die Semantik von da-Sätzen genauer betrachten.

Gehen wir zunächst vom indefiniten ( $=$ bezüglich Definitheit unmarkierten) weil-Satz aus. Weil seine Lampe noch brennt bedeutet: ,es ist der Fall, dass seine Lampe noch brennt, und das ist Ursache für p', wobei p der im Hauptsatz beschriebene Sachverhalt ist.

Betrachten wir nun den entsprechenden da-Satz. $D a$ bringt über die Bedeutung des weil-Satzes hinaus die Definitmarkierung ein, durch die die Ursache als bekannt gekennzeichnet wird. Da seine Lampe noch brennt bedeutet also: ,es ist bekanntlich der Fall, dass seine Lampe noch brennt, und das ist Ursache für p'. Dies besagt zum einen, dass es der Fall ist, dass die Lampe noch brennt. Somit hat der da-Satz eine dispositionelle Lesart, in der zwei Sachverhalte kausal verknüpft werden. Zum anderen aber kann man aus bekanntlich der Fall folgern: ,es ist wahr, dass seine Lampe noch brennt, und das ist Evidenz für p'.

Diese Folgerung bildet den Übergang von der dispositionellen zur epistemischen Lesart von (30b). Sie ist alltags- und formallogisch gerechtfertigt: Was bekanntlich der Fall ist, kann mit Fug und Recht als wahr behauptet werden. Die Wahrheit einer solchen Behauptung wird durch die Bekanntheit der Faktizität des behaupteten Sachverhaltes sichergestellt. Damit aber wird zugleich das Sachverhaltskomplement von da umkategorisiert zu einem Propositionskomplement, denn was der Fall ist, ist immer ein Sachverhalt, was wahr ist, kann dagegen nur eine Proposition sein. Es ist also tatsächlich die Definitheitskomponente von 
$d a$, die die epistemische Lesart von $d a$-Sätzen im Vorfeld möglich macht, und zwar auf semantisch-kompositionellem Wege (,der Fall' + ,bekanntlich' $\rightarrow$,wahr'), ohne dass solche Nebensätze kommunikative Minimaleinheiten mit eigener Fokus-Hintergrund-Gliederung und eigenem Hauptakzent bilden müssen. ${ }^{4}$

\subsection{Modale Umgebung}

Abschließend möchte ich noch auf einen weiteren wichtigen Faktor hinweisen, der die Lesart von Kausalverknüpfungen beeinflusst, nämlich die Modalität der Satzumgebung. Sie wird unter anderem durch den Satzmodus, durch Modalverben und durch Modalpartikeln erzeugt (vgl. Kratzer 1991: 639f.). SweEtser (1990: 78) beobachtet, dass kausale Verknüpfungen nicht anders als deontisch-illokutionär interpretiert werden können, wenn der Haupt- bzw. Vordersatz im interrogativen oder imperativen Satzmodus steht wie in (32). Wenn epistemische Ausdrücke wie ich glaube o.ä. im Kontext vorhanden sind wie in (33), ist eine epistemische Lesart naheliegend (ebd.: 80):

(32) Was machst du heute abend? Ich hab nämlich Kinokarten gekauft.

(33) Ich glaube, Thomas ist krank, weil ich ihn schon seit Tagen nicht gesehen habe.

4 Dem Übergang von der dispositionellen zur epistemischen Lesart kann, je nach Kontext, eine Korrespondenz-, eine Kohärenz- oder eine Konsens-Definition der Wahrheit zugrundeliegen. Alle drei Definitionstypen gehen in den alläglichen Wahrheitsbegriff ein (vgl. Gethmann 1978). - An dieser Stelle ist auch der Hinweis angebracht, dass der beschriebene Übergang nur zu epistemischen, nicht aber allgemein zu modalen Lesarten führt. So kann aus der bekannten Faktizität eines Sachverhaltes zwar auf die Wahrheit einer Proposition, nicht aber auf die Erwünschtheit einer Handlung geschlossen werden. Deshalb sind da-Sätze im Vorfeld nur epistemisch, nicht aber deontisch-illokutionär interpretierbar. Vgl. dazu die Beispiele (20a)-(20d) in Abschnitt 4.1, an denen gezeigt wurde, dass eine deontische Lesart bei syntaktischer Integration nicht in Frage kommt. 
Eine genauere Analyse modaler Satzumgebungen und ihres Einflusses auf die Konnektor-Lesart muss auf eine spätere Gelegenheit verschoben werden. Ich möchte aber darauf hinweisen, dass Korpusbelege für Kausalverknüpfungen, bei denen sich modale Konnektor-Lesarten aufdrängen, zuallermeist neben geeigneten syntaktischen, prosodischen und informationsstrukturellen Bedingungen auch solche modalen Markierungen enthalten. Um den genauen Einfluss der Faktoren auf die Konnektor-Lesart bestimmen zu können, ist es notwendig, sie getrennt voneinander zu untersuchen, was am besten durch Manipulation von Laborbeispielen geschehen kann. Deshalb habe ich die meisten Beispiele für den vorliegenden Aufsatz selbst gebildet und nur wenige Originalbelege verwendet.

\section{Zusammenfassung}

Abschließend gebe ich einen Überblick über die fünf Hauptthesen, die in diesem Aufsatz entwickelt wurden:

1. Kausalverknüpfungen sind Bestandteile der kausalen Textkohärenz. Typischerweise müssen sie vom Textinterpreten selbständig durch Inferenzen hergestellt werden. Nur wenn der Sprecher es für relevant hält, den Interpreten auf eine ganz bestimmte kausale Interpretation festzulegen, werden sie explizit im Text kodiert.

2. Explizite Kausalverknüpfungen werden hauptsächlich durch lexikalische Mittel hergestellt, und zwar durch Präpositionen, Konjunktionen (Subjunktoren, Konjunktoren) und Adverbien. Präpositionen und Konjunktionen stellen syntaktische und semantische Verknüpfungen her, Adverbien nur semantischreferentielle.

3. Kausalverknüpfungen zwischen außersprachlichen Ereignissen und Kausalverknüpfungen zwischen Sätzen sind ganz unterschiedliche Dinge. Für erstere interessieren sich Physik und Philosophie; für letztere interessiert sich die Sprachwissenschaft. 
4. Kausale Satzverknüpfungen können nicht-modal (dispositionell) oder modal (epistemisch oder deontisch-illokutionär) interpretiert werden. Die dispositionelle Deutung ist als der Normalfall anzusehen. Modale Lesarten sind demgegenüber markiert. Sie sind dadurch gekennzeichnet, dass der Konnektor Skopus über die modalen Operatoren der Teilsätze hat.

5. Modale Konnektor-Lesarten werden durch vier syntaktisch-semantische Faktoren begünstigt: syntaktische Desintegration des Kausalausdrucks, getrennte Akzentuierung der Teilsätze, Definitheit der Ursache und Modalitätsmarker in der Satzumgebung. Jeder einzelne dieser Faktoren macht für sich genommen modale KonnektorLesarten wahrscheinlicher. Je mehr Faktoren zusammenwirken, desto unwahrscheinlicher werden dispositionelle Lesarten.

\section{Literaturverzeichnis}

BLÜHDORN, Hardarik (2002). „Rauminformation und Demonstrativität. Am Beispiel des Deutschen". In: Deutsche Sprache 30, 252-275.

BLÜHDORN, Hardarik (2003). „Zur Semantik der Konjunktion als. Paradigmatische und syntagmatische Aspekte". In: Linguistik online 13, 11-53 (http://www.linguistik-online.de/13_01/bluehdorn.pdf).

BLÜHDORN, Hardarik (2006). ,Zur Semantik von Numerus und Zählbarkeit im Deutschen". In: Breindl, Eva / Gunkel, Lutz / Strecker, Bruno (Hg.). Grammatische Untersuchungen, Analysen und Reflexionen. Festschrift für Gisela Zifonun. Tübingen, Narr, 53-77.

BLÜHDORN, Hardarik (i.E.). „Epistemische Lesarten von Satzkonnektoren - Wie sie zustande kommen und wie man sie erkennt". In: POHL, Inge (Hg.). Semantik vs. Pragmatik oder Semantik und Pragmatik? Frankfurt/Main, Lang (erscheint 2007).

BürIng, Daniel (1997). The Meaning of Topic and Focus. The 59 $9^{\text {th }}$ Street Bridge Accent. London, Routledge.

Duden (1998). Grammatik der deutschen Gegenwartssprache, 6. Aufl., Mannheim, Dudenverlag. 
Gethmann, Carl Friedrich (1978). „Wahrheit”. In: Braun, Edmund / RADERMACHER, Hans (Hg.). Wissenschaftstheoretisches Lexikon. Graz, Styria, 645648.

Haldiday, M.A.K. / Hasan, Ruqaiya (1976). Cohesion in English. London, Longman.

JACOBS, Joachim (1984). „Funktionale Satzperspektive und Illokutionssemantik". In: Linguistische Berichte 91, 25-58.

Keller, Rudi (1995). „The epistemic weip’. In: STEIN, Dieter / Wright, Susan (Hg.). Subjectivity and subjectivisation. Linguistic perspectives. Cambridge, University Press, 16-30.

KONDAKOW, N.I. (1983). Wörterbuch der Logik (deutsch von Erhard Albrecht und Günter Asser). Leipzig, VEB Bibliographisches Institut.

Kratzer, Angelika (1991). „Modality”. In: von STECHow, A. / WunderLICH, D. (Hgg.). Semantik. Ein internationales Handbuch der zeitgenössischen Forschung, Berlin, de Gruyter, 639-650.

Moroni, Manuela (2006). Modalpartikeln an der Schnittstelle zwischen Syntax und Prosodie. Doktorarbeit. Verona, Università di Verona / Mannheim, Institut für Deutsche Sprache.

PAsch, Renate (1997). „Weil mit Hauptsatz - Kuckucksei im denn-Nest”. In: Deutsche Sprache 25, 252-271.

Pasch, Renate / Brausse, Ursula / BreindL, Eva / Wassner, Ulrich Hermann (2003). Handbuch der deutschen Konnektoren. Linguistische Grundlagen der Beschreibung und syntaktische Merkmale der deutschen Satzverknüpfer, Berlin, de Gruyter.

PfeIfer, Wolfgang et al. (1989). Etymologisches Wörterbuch des Deutschen, 3 Bde., Berlin (Ost), Akademie.

RedDER, Angelika (1990). Grammatiktheorie und sprachliches Handeln: „denn” und „,da”, Tübingen, Niemeyer.

Stegmüller, Wolfgang $(1960=1974)$. „Das Problem der Kausalität”. In: STEGMÜLLER, Wolfgang (1974). Aufsätze zur Wissenschaftstheorie, Darmstadt, Wissenschaftliche Buchgesellschaft, 1-20. (Nachdruck aus E. TOPITsch (Hg.) (1960). Probleme der Wissenschaftstheorie. Festschrift für Victor Kraft, Wien, Springer, 171-190.) 


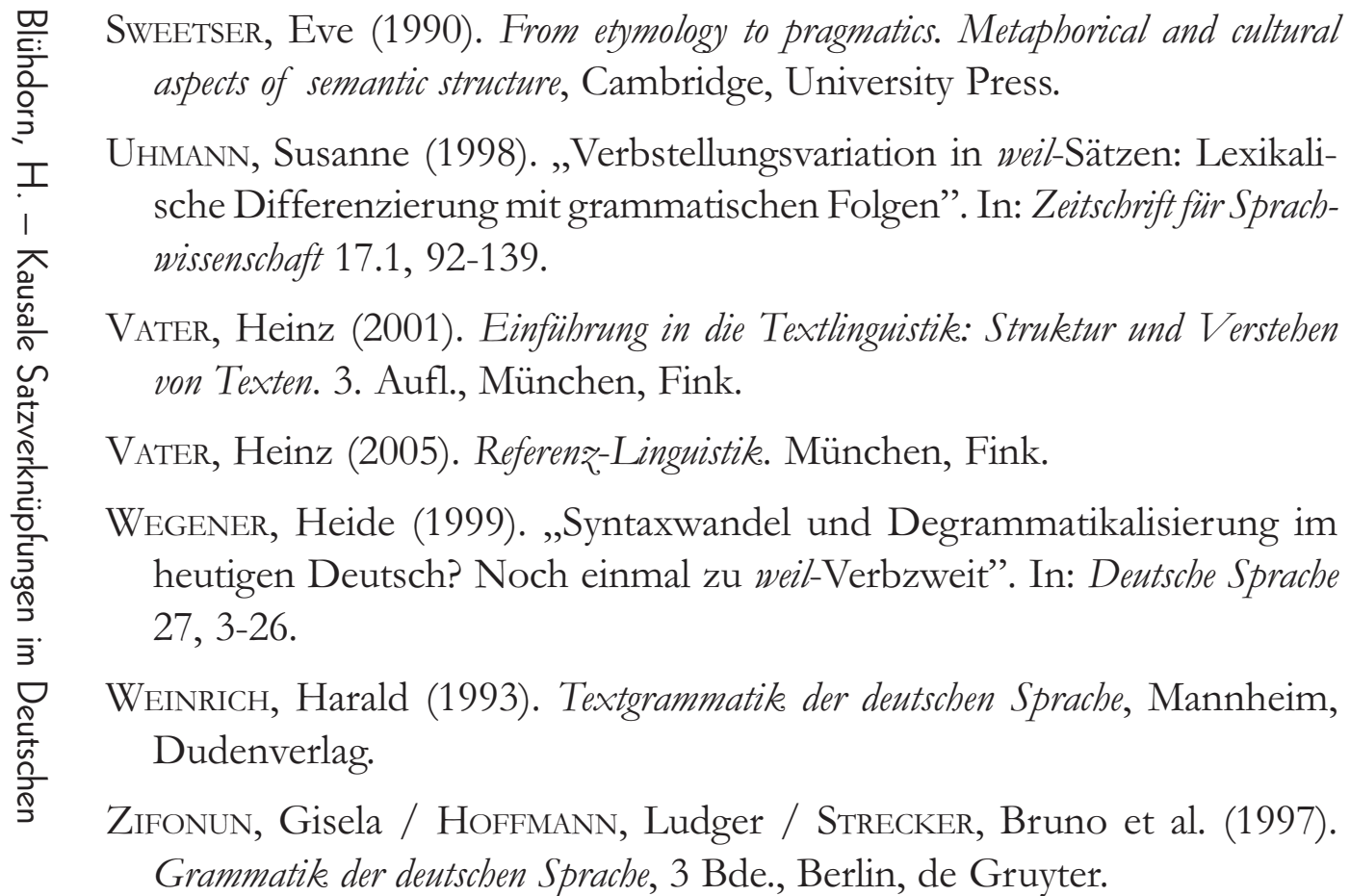

